\title{
Effect of Filler Loading, Geometry, Dispersion and Temperature on Thermal Conductivity of Polymer Nanocomposites
}

\author{
Addis Tessema ${ }^{\mathrm{a}}$, Dan Zhao ${ }^{\mathrm{b}}$, Joseph Moll ${ }^{\mathrm{b}}$, Shansan $\mathrm{Xu}^{\mathrm{c}}$, , Ronggui Yang ${ }^{\mathrm{c}}$, Chen Li ${ }^{\mathrm{a}}$, Sanat K. \\ Kumar $^{\mathrm{b}}$ Addis Kidane ${ }^{1 \mathrm{a}}$, \\ a Department of Mechanical Engineering, University of South Carolina, 300 Main Street, Columbia, South Carolina 29208, United States \\ ${ }^{\mathrm{b}}$ Department of Chemical Engineering, Columbia University, 500 West 120th Street, New York, New York 10027, United States \\ ${ }^{c}$ Department of Mechanical Engineering, University of Colorado, 1111 Engineering Dr., Boulder, CO 80309, United States
}

\begin{abstract}
Using a unidirectional heat transfer apparatus, the roles of nanoparticle geometry, loading, dispersion and temperature on the thermal conductivity of polymer nanocomposites are investigated. The polymer nanocomposites (PNC) consist of epoxy matrices filled with silica nanopowder and carbon nanotubes, respectively, as well as poly (2-vinylpyridine) (P2VP) matrices loaded with silica nanoparticles. First, it is shown that thermal conductivity generally increases with nanofiller loading. These results are also reasonably described by the three phase Lewis-Nielsen or Halpin-Tsai analytical models. More importantly, it has been also demonstrated that the thermal conductivity of the polymer nanocmposites greatly depends on the dispersion state of the nanofillers. Furthermore, the effect of temperature on the thermal behavior of PNCs is briefly discussed. These results emphasize the important role of nanoparticles content and dispersion state on the thermal characteristics of polymer nanocomposites, which can be used to design composite materials with tunable thermal behavior.
\end{abstract}

\footnotetext{
${ }^{1}$ Corresponding Author - Address: Department of Mechanical Engineering, 300 Main Street, Columbia, SC 29208 Tel: +1 (803) 777-2502 | Fax: +1 (803) 777-0106 | E-mail: kidanea@cec.sc.edu
} 
Keywords: Thermal conductivity, Polymer nanocomposites, Nanoparticle dispersion, Carbon nanotubes

\section{Introduction}

Polymeric materials have been widely used for electronic packaging, high voltage transformers, cable terminations, and bushings or insulation for X-ray tubes [1-6]. Under these applications, the polymers are simultaneously exposed to mechanical and thermal loadings, and their thermal behavior plays a vital role in the overall performance of the entire system. In order to tune or improve the thermal conductivity of these macromolecules, nano sized inorganic particles have been incorporated into the polymer matrices[7-9]. Previous works suggested that the effective thermal conductivity of such composite materials is influenced by the thermal properties of the constituents $[7,9,10]$, the thickness and characterstics of the interfacial layer [2,11,12], the filler's geometry/aspect ratio [13] and filler volume fraction [2-4,8,13-16]. However, to our knowledge, there is no systematic study to understand the combined effect of particle loading, geometry, dispersion state and temperature on the thermal conductivity of PNC.

In nanoparticle (NP) filled composites, the major issue is the clustering of the filler particles due to strong Van der Waals inter-NP forces. The extent of clustering or agglomeration has a direct dependence on the effective amount of filler and filler geometry. The tendency for agglomeration grows with the particle loading and/or the aspect ratio of the particle. Different mechanical and chemical techniques have been developed to overcome agglomeration over the years, but it still persists to some degree in highly loaded composite materials [17]. Recently, a polymer surface treatment using chemical solvent provided promising results in terms of dispersing the nanoparticles in polymers [17-22]. 
In this paper, we investigate the effect of nanofillers on the thermal conductivity of PNC, by accounting for the weight fraction, particle geometry and distribution of the fillers and the working temperature. Two types of fillers, higher aspect ratio carbon nanotubes and spherical silica, respectively, are used to characterize the role of particle geometry or aspect ratio. The effect of particle dispersion states is also investigated based on two different systems: carbon nanotube (CNT) samples with or without ultrasonication and silica-P2VP cast from different solvents. In addition, the effect of temperature on the thermal conductivity of the nanocomposites is also presented.

Analytical models are also used to model and understand these experimental findings. In the last few decades, different models have been proposed by implementing various analytical and semiempirical techniques to estimate the mechanical and thermal property of composite materials. The series model, parallel model, the Lewis-Nielson (L-N), Maxwell Eucken-EMA (ME-EMA), Hamilton and Cheng-Vachon, and Halpin-Tsai models are a few proposed to determine the thermal conductivity of nanocomposites [2,3,11,23-25]. Most of these models take filler geometry, weight ratio of the filler, particle distribution, thermal conductivity of both the matrix and the filler, and interface thermal resistance (Kapitaz resistance) at the junction between the filler and the matrix into account. In this work, the three phase Lewis-Nielson and the modified Halpin-Tsai models are adopted due to their flexibility in allowing different material geometries, namely the particle, the matrix polymer and any interfacial material.

\section{Materials and Methods}

\subsection{Materials}

Three different types of PNCs, namely epoxy reinforced with silica particles (Type 1), epoxy reinforced with carbon nanotubes (CNTs) (Type 2), and silica-poly(2-vinylpyridine) [P2VP] 
nanocomposites solvent cast from either methyl ethyl ketone (MEK) or pyridine solutions (Type 3) are considered. The weight fraction of the NPs was varied systematically in each class of PNC. The base matrix material used for Type 1 and Type 2 nanocomposites is Bisphenol-A epoxy (Buehler Epothin 2 Resin, Part-A) with compatible hardener, recommended by the manufacturer (Bueler Epothin 2 Hardener, Part-B). The nanoparticles used for Type 1 materials are spherical silica nanopowders with a primary particle size of 10 to $20 \mathrm{~nm}$ (BET) and 99.5 trace metal basis purchased from Sigma Aldrich. The nanoparticles used for Type 2 materials are tubular 95\% pure CNTs, purchased from NanoLab Inc, with an outer diameter of $30 \pm 15 \mathrm{~nm}$ and length of 5 to 20 microns. The silica NPs used in Type 3 samples were donated by Nissan Chemical Industries, which were incorporated into a poly (2-vinylpyridne) ( $\mathrm{P} 2 \mathrm{VP})$ matrix $\left(M_{\mathrm{w}}=\right.$ $\left.105 \mathrm{Kg} / \mathrm{mol}, M_{\mathrm{w}} / M_{\mathrm{n}}=1.08\right)$ purchased from polymer source Inc. The antioxidant Irganox 1010 (Ciba Specialty Chemicals, now BASF Switzerland) was used to avoid thermal degradation during annealing at $150{ }^{\circ} \mathrm{C}$ for this last class of materials.

\subsection{Nanocomposite preparation}

For Type 1 nanocomposites, the as-received epoxy resin (Part-A) and silica nanopowder were weighed to obtain the desired NP weight fraction. The constituents were mixed mechanically in a glass beaker until the mixture turned to a uniform color. Then the mixture was ultrasonicated to obtain a uniform distribution of the filler in the matrix. The ultrasonication is made at $24 \%$ of the maximum amplitude with pulse mode of $2 \mathrm{sec}$ ON and $1 \mathrm{sec}$ OFF for 30 mins. In order to maintain the temperature uniform during ultrasonication process the beaker was kept in an ice bath. The mixture was then kept in a vacuum chamber for about 10-30 mins and the air trapped during mechanical and ultrasonic mixing was sucked out. Afterwards, the right amount of hardener was added to the mixture (i.e. 1:2 ratio to the epoxy) and mixed thoroughly. The 
mixture was brought back to and kept in the vacuum chamber again for a few minutes to remove any further trapped air. Following this the mixture was poured into silicon rubber molds that are coated with a release agent, and placed in a controlled hood to cure for 48 hrs. Finally, disc shaped samples with a diameter of $25.4 \mathrm{~mm}$ and a thickness of $1 \mathrm{~mm}-1.5 \mathrm{~mm}$ were prepared and the sample surfaces were polished with different grit size sand papers.

In Type 2, nanocomposite (CNT-epoxy) samples were also prepared following a similar procedure except for some changes in the ultrasonication parameters. CNT particles have a strong tendency to cluster together and usually a stronger shaking force is required to disperse them well. Ultrasonic waves are used to break the clustering bond, however, since CNTs have the ability to change their chemical form due to the sonic wave, the wave amplitude must be tuned, and the ultrasonication timing must be adjusted to keep the mixing temperature low. Considering these effects, the CNT-epoxy samples are ultrasonicated at $40 \%$ of the maximum amplitude with pulse mode of $5 \mathrm{sec}$ ON and 10sec OFF for 1 hour. To probe the effect of particle distribution, samples were made following the same procedure but without using the ultrasonication.

The detailed fabrication process of Type 3 nanocomposites can be found elsewhere [18]- we briefly summarize the procedure here for completeness. First, P2VP and silica NPs with the appropriate weight ratio was mixed in either MEK or pyridine. The resulting solutions were vortex-shaken for $2 \mathrm{~h}$ and probe-ultrasonicated for $3 \mathrm{~min}$. The solutions were then poured into a PTFE Petri dish, air dried in a fume hood for 5 days. To remove the residual solvent, the as-cast samples were then annealed at $150{ }^{\circ} \mathrm{C}$ for $24 \mathrm{~h}$ under vacuum.

\subsection{Thermal conductivity characterization}


The thermal conductivity of all samples was measured using a linear unidirectional heat transfer apparatus shown in Figure 1. The apparatus has six thermocouples to monitor the temperature flow through and across the cross section of the specimen. This device was set in an arrangement in which there is an electrical heat source at the top and a constantly flowing water sink at the bottom. The specimen was sandwiched between the two $25.4 \mathrm{~mm}$ diameter brass rods. The top rod was connected with a heater while the bottom rod was connected to constantly running water. The sectioned schematic diagram of the experimental setup is shown in Figure 1, where T1-T6 represents the locations of the six thermocouples and $\mathrm{T}_{\mathrm{H}}$ and $\mathrm{T}_{\mathrm{C}}$ represents the top and lower surface of the specimen.

The heat source and the associated temperatures can be controlled by adjusting the input voltage. In this work, experiments were conducted at three different settings corresponding to $5 \mathrm{~V}, 6.5 \mathrm{~V}$ and $8 \mathrm{~V}$ source voltages. During the test, once steady-state was achieved, the temperatures were measured using the thermocouples. The thermal conductivity of each specimen can be calculated from the temperature records and geometries, as given by Eq.1.

$$
K_{e}=K_{\text {Brass }}\left[\frac{\left(\Delta X_{1-3}\right) *\left(T_{1}-T_{3}\right)}{\left(\Delta X_{\text {Specimen }}\right) *\left(T_{H}-T_{T}\right)}\right]
$$

Where $K_{\text {Brass }}$ is the thermal conductivity of the brass conductors, $\Delta \mathrm{X}_{\text {Specimen }}$ is the thickness of the test material; $\Delta X_{1-3}$ is the distance between the thermocouples $T_{1}$ and $T_{3} . T_{H}$ and $T_{C}$ are temperature at the top and bottom surface of the specime, obtained by extrapolating temperature readings form $T_{1}-T_{3}$ and $T_{4}-T_{6}$ respectively.

\section{Analytical Method}




\subsection{Three phase Lewis-Nielson (LN) Model}

The three phase Lewis-Nielson (LN) model is adapted from the Halpin-Tsai (HT) mechanical model. It is a semi-empirical equation that considers the effect of particle size, shape of the particle (aspect ratio), weight ratio of the particle and the critical or maximum packing of particles [3]. It also accounts for the interface layer resistance by taking the layer as a third phase within the composite. The effective thermal conductivity of the composite can be given as:

$$
K_{e}=K_{m}\left[\frac{\left(1+\varepsilon^{*} \eta^{*} \varphi_{c p}\right)}{\left(1-\phi^{*} \eta^{*} \varphi_{c p}\right)}\right]
$$

Where, $\phi=1+\left[\frac{\left(1-\varphi_{m}\right)}{\left(\varphi_{m}{ }^{2}\right)}\right] \varphi_{c p}, \eta=\left[\frac{\left(K_{i}-K_{m}\right)}{\left(K_{i}+K_{m} * \varepsilon\right)}\right], \varepsilon$ is the aspect ratio, $\varphi_{\mathrm{m}}$ is the maximum packing factor and $\mathrm{K}_{\mathrm{i}}$ is the combined thermal conductivity of the interface and particle respectively which is calculated as,

$$
K_{i}=1 /\left[\left[\frac{\varphi_{p}}{K_{f}\left(\varphi_{p}+V_{i}\right)}\right]+\left[\frac{\varphi_{i}}{K_{m}\left(\varphi_{p}+\varphi_{i}\right)}\right]\right]
$$

$\mathrm{K}_{\mathrm{m}}$ and $\mathrm{K}_{\mathrm{f}}$ are the thermal conductivity of the matrix and the particle, respectively, $\varphi_{\mathrm{p}}$ is the volume fraction of the particles, and $\varphi_{\mathrm{i}}$ is the volume fraction of the interface layer which is obtained as shown from Eq.4:

$$
\varphi_{i}=\varphi_{p}\left[\frac{\left(r_{p}+t\right)^{3}}{\left(r_{p}\right)^{3}}-1\right] \text { and } \varphi_{c p}=\varphi_{p} * \delta
$$

$\delta=\left(1+t / r_{p}\right)^{3}$ and $\mathrm{r}_{\mathrm{p}}$ is the radius of the particle, $\varphi_{\mathrm{cp}}$ is the combined volume fraction of particle and interface layer. The effective thermal conductivity $\left(\mathrm{K}_{\mathrm{e}}\right)$ obtained from this analytical model is shown in Fig. 2 and 3. 


\subsection{Halpin-Tsai (HT) Model}

The Halpin-Tsai model is a semi-empirical model usually used to predict the elastic constants $[2,23,24]$ especially for randomly oriented and high aspect fillers, such as for carbon nanotube reinforced composites. This model also considers different factors, such as the geometry of the filler, the properties of the filler and the matrix, volume fraction of the filler and filler distribution. In our study, the model was adopted and implemented to determine the effective thermal conductivity of nanocomposites made with CNT fillers. The model incorporates two parts as shown in Eq. 5, one for transverse and one for longitudinal thermal conductivities,

$$
k_{e}=\frac{3}{8}\left[1-2\left(\frac{l}{d}\right) *\left[\frac{\frac{k_{f}}{k_{m}}-\frac{d}{4 t}}{\frac{k_{f}}{k_{m}}+\frac{l}{4 t}}\right] \phi_{f}\right] *\left[1-\left[\frac{\frac{k_{f}}{k_{m}}-\frac{d}{4 t}}{\frac{k_{f}}{k_{m}}-\frac{d}{2 t}}\right] \phi_{f}\right] * K_{f}+\frac{5}{8}\left[1-2\left[\frac{\frac{k_{f}}{k_{m}}-\frac{d}{4 t}}{\frac{k_{f}}{k_{m}}+\frac{l}{2 t}}\right] \phi_{f}\right] *\left[1-\left[\frac{\frac{k_{f}}{k_{m}}-\frac{d}{4 t}}{\frac{k_{f}}{k_{m}}+\frac{d}{2 t}}\right] \phi_{f}\right] * K_{f} \ldots \ldots \ldots \ldots . .(5)
$$

Where $l, t$ and $d$ are the length, thickness and diameter of the CNT filler, $K_{f}$ and $K_{m}$ are thermal conductivities of the filler and the matrix respectively and $\phi_{f}$ is the volume fraction of the filler CNT.

\section{Results and Discussion}

\subsection{Effect of Particle Loading}

Figure 2, presents the effective thermal conductivity of $\mathrm{SiO}_{2}$-epoxy nanocomposites (type-1) as a function of particle content. As shown in the figure 2, the effective thermal conductivity $\left(K_{\mathrm{e}}\right)$ of the PNCs increases with an increase in the weight fraction of silica NPs. This should be attributed to the much higher thermal conductivity of silica materials $(2 \mathrm{~W} / \mathrm{mK})$ compared to that of the epoxy matrix $(0.15 \mathrm{~W} / \mathrm{mK})$. From Figure 2 , it is also clear that the increment in $K_{\mathrm{e}}$ is not linear with the particle content, and qualitatively, it can be divided into three regions. In the first region at low particle loading, a rapid increase ( $\sim 35 \%$ increment with inclusion of $2 \mathrm{wt} \%$ silica) 
in the thermal conductivity was observed, where the nanoprticles were expected to be uniformly dispersed. In the second region, as the weight fraction of particles increases the slope of the increment in $K_{\mathrm{e}}$ is reduced ( $\sim 14 \%$ increment from $2 \mathrm{wt} \%$ to $4 \mathrm{wt} \%$ ), presumably due to particle clustering as more particles are added into the matrix. Going further (third region), when the filler content increased to larger than $6 \mathrm{wt} \%$, the increment in $K_{\mathrm{e}}$ rose again. In the third region, higher particle packing is expected, and this packing brings a continuous particle to particle heat channel. As a result, the thermal barrier effect due to the local clustering is over dominated by the particle to particle channeling, and hence the thermal conductivity increases in region 3 . Figure 2 also provides the prediction of $K_{\mathrm{e}}$ as a function of particle loading by both the modified Halpin-Tsai and the Three phase Lewis-Nielsen models. Clearly, the Three phase Lewis-Nielsen model agrees much with the experimental results, with a maximum variation of $8 \%$.Furthermore, the trend in the rate of increment in $K e$ for the three different regimes is quite well captured by this model. In contrast, the modified Halpin-Tsai model deviates appreciably from the experimental values, with the discrepancy increasing with the increase in the particle concentration. We believe that the failure of accounting for the interface effect in the Halpin-Tsai model should be the main reason for such a high discrepancy. Based on these results, we suggest that the Three phase Lewis-Nielson model is reasonably appropriate for PNCs comprised of NPs with a spherical geometry or low aspect ratio.

The Ke of CNT-epoxy nanocomposites (Type-2) as a function of particle loading is shown in Figure 3. Similar to the silica filled system, the Ke of the CNTs filled PNCs increases monotonically with the amount of CNT incorporated. Due to the high thermal conductivity of the CNT fillers, the Ke of the composite materials increases dramatically with even a small amount of CNT added. For example, the Ke increases by $\sim 32 \%$ with the addition of only 0.1 wt $\%$ CNT 
into the plain epoxy and $\sim 80 \%$ for the $0.4 \mathrm{wt} \%$ sample. At higher particle content, the rate of increment in Ke becomes smaller, which is the result of the particle agglomeration at high particle loadings.

Figure 3 also depicts the analytical prediction of $K_{\mathrm{e}}$ as a function of particle loading for CNT filled PNCs from the Lewis-Nielson and the modified Halpin-Tsai models, respectively. The Lewis-Nielson model shows an almost linear dependence on weight ratio in good agreement with experimental results at higher NP content. The modified Halpin-Tsai model, on the other hand, shows a much better prediction for experimental results across the whole range of compositions; the maximum deviation obtained from this model is about $5 \%$. In general both models have shown appreciable approximations and agreement with the experimental result for CNT-epoxy composites, this indicates that the Lewis-Nielson model can also be effecitvely used for large aspect ratio fillers.

\subsection{Effect of Particle Dispersion}

Previous studies show that the materials properties specifically the mechanical behavior of PNCs depends strongly on the spatial organization of the added NPs. It is generally suggested that uniform particle dispersion is preferred to maximize the particle/polymer interfacial interaction and improve the mechanical properties of the nanomcoposites. The effect of particle distribution on the effective thermal conductivity of the nanocomposites is investigated.

We have examined two different PNC systems: one is the CNTs reinforced epoxy and the other is composed of silica NPs and P2VP matrices. In the former case, the spatial distribution of CNTs is manipulated by the ultrasonication. As shown in Figure 4, prior to ultrasonication, the CNTs are strongly agglomerated in the matrices, with typical cluster sizes of $\sim$ microns (Figure $4 \mathrm{a}$ and $4 \mathrm{~b}$ ). In contrast, with ultrasonication, the large clusters are broken up and the dispersion 
of CNTs is greatly improved; even individual particles can be found dispersed in the matrix (Figure $4 \mathrm{c}$ and $4 \mathrm{~d}$ ).

In another system, the silica NPs are added into a P2VP matrix, in which the distribution of silica NPs can be facilely controlled by the choice of the casting solvent. Our previous work [16] has shown that, in the films cast from MEK, a boundary layer of P2VP forms at the NP surface. The resulting "hairy" particles are sterically stabilized against agglomeration (Figure 5b). In contrast, in pyridine, P2VP does not adsorb onto the silica surface. Thus the bare silica particles are aggregated into fractal clusters due to polymer-induced depletion attraction (Figure 5a). We have also demonstrated that the aggregated morphology of pyridine cast samples cannot be completely annealed away due to the strong Van der Waals interaction between the particles.

With this capability we examined the effect of particle organization on the thermal behavior of the resulting composites. As shown in Figure 6, for the non-ultrasonicated samples, the effective thermal conductivity of the nanocomposites increased at NP loading at lower CNT content, but decreases as the weight fraction of the CNT increases. In contrast, the well-mixed ultrasonicated samples show a monotonic increase of thermal conductivity with loading. It is believed that the presence of agglomerated clusters causes the scattering of heat carrier phonons and reduces the flow of heat in a uniform direction, and as a result, the thermal conductivity reduces. In addition, the temperature discontinuity created by local high thermal conductivity of the agglomerated particle imposes non-uniform heat flow, which could potentially reduce the effective thermal conductivity of the nanocomposites.

For the other sets of samples, figure 7 compares the thermal conductivity of Type 3 PNCs cast from these two different solvents. First, as expected, for PNCs cast from both solvents, the effective thermal conductivity increases with the weight fraction of the silica nanofillers. 
Interestingly, at low silica loadings (less than $40 \mathrm{wt} \%$ ), the films cast in MEK generally transport heat more efficiently than those cast in pyridine. This can be reconciled by the fact that the average inter-particle distance is significantly smaller in the case of using MEK as the solvent where the NPs are well distributed. As a result, the phonons can propagate more easily than in the agglomerated case, making their transport in a more homogeneous way.

In contrast, when the particles are agglomerated, as the phonons prefer to go through the particle phase, the length of the polymer phase involved in phonon propagation becomes longer, leading to lower conductivity. Furthermore, at high silica content, e.g., $45 \mathrm{wt} \%$ and $50 \mathrm{wt} \%$, the values of thermal conductivity for samples cast from different solvents nearly converge, probably because the particle phase percolates. In other words, the particle morphology evolves to be quite similar in these two cases [19], leading to no significant difference in thermal conductivity.

\subsection{Effect of Temperature}

Prior to understanding the effect of temperature on the thermal conductivity of the composites, we first examine the glass transition temperature $(\mathrm{Tg})$ of these materials as a function of silica loading by using Differential Scanning Calorimetry (DSC). As shown in Figure 8, the Tg increases with the silica weight fraction, indicating favorable interaction between the polymer and the particle. Also note that the magnitude of $\mathrm{T}_{\mathrm{g}}$ increase is small, i.e., less than $5 \mathrm{~K}$, as observed in other works $[1,26]$.

The effective thermal conductivity as a function of temperature for epoxy-Silica nanocomposites is shown in Figure 9. Note that each specimen has been tested at three different temperatures. At temperatures close to the glass transition, it is expected that the polymer chains are highly mobile. This should increase the thermal conductivity by allowing more phonon vibration and heat transportation. This effect can be seen in Figure 9, for pure epoxy and lower weight ratio 
filler, where the effective thermal conductivity increases linearly with temperature. Clearly, as temperature increases, the resistance for the phonon propagation reduces, thus leading to faster heat transport.

As shown in Figure 9, at silica content less than 4 wt \%, the thermal conductivity increases with the temperature, and almost remain constant for $4 \mathrm{wt} \%$. Interestingly, at $6 \mathrm{wt} \%$, the thermal conductivity first increases up to certain temperature and starts to drop. This indicates that there is a possibility that the presence of higher filler content within the polymer intiates phonon scattering at temperature close to the $\mathrm{T}_{\mathrm{g}}$.

\section{Summary}

The effective thermal conductivity of particle filled nanocomposites is examined both experimentally and numerically. The effects of particle loading, geometry and distribution, as well as the operating temperature on the effective thermal conductivity of nanocomposites are explored. From the current experimental and numerical results, the following points can be summarized:

- The thermal conductivity of the nanocomposites increases with nanofillers added, which depends strongly on the thermal properties of the fillers used.

- Dispersion of the NPs in the polymeric matrices is critical to the thermal conductivity of the resulting PNCs.

- The thermal conductivity of the nanocomposites with well dispersed particles increases with increasing the particles weight ratio. While, for non-uniform distribution of particles, the thermal conductivity decreases as weight ratio increases. This leads to the deduction that NP agglomeration in non-dispersed particles might have decreased the resistance for heat transport. 
- The effective thermal conductivity of the nanocomposites with lower silica content, increases with testing temperature.

It was clearly demonstrated that for composites with spherical particles the Lewis - Neilson model is more appropriate, while both the Lewis-Neilson and the Halpin-Tsai models can be effectively used for nanocomposites with large aspect ratio particles.

\section{Acknowledgement}

The financial support provided by NSF under Grant No EEC-1342379, is gratefully acknowledged. 


\section{Reference}

[1] M. Iqbal, G. Mamoor, T. Bashir, M. Irfan, M. Manzoor, A Study of Polystyrene-Metal Powder Conductive Composites, J. Chem. Eng. 25 (2011) 5-8.

[2] C.W. Nan, R. Birringer, D.R. Clarke, H. Gleiter, Effective thermal conductivity of particulate composites with interfacial thermal resistance, J. Appl. Phys. 81 (1997) 66926699. doi:10.1063/1.365209.

[3] R. Kochetov, a V Korobko, T. Andritsch, P.H.F. Morshuis, S.J. Picken, J.J. Smit, Modelling of the thermal conductivity in polymer nanocomposites and the impact of the interface between filler and matrix, J. Phys. D. Appl. Phys. 44 (2011) 395401 (12pp).

[4] J. Kratochvíla, A. Boudenne, I. Krupa, Effect of filler size on thermophysical and electrical behavior of nanocomposites based on expanded graphite nanoparticles filled in low- density polyethylene matrix, Polym. Compos. 34 (2013) 149-155.

[5] M. Jouni, A. Boudenne, G. Boiteux, V. Massardier, B. Garnier, A. Serghei, Electrical and thermal properties of polyethylene/silver nanoparticle composites, Polym. Compos. 34 (2013) 778-786.

[6] E.Ç. Bayramoglu, Thermal properties and stability of n- octadecane based composites containing multiwalled carbon nanotubes, Polym. Compos. 32 (2011) 904-909.

[7] K. Yang, M. Gu, Enhanced thermal conductivity of epoxy nanocomposites filled with hybrid filler system of triethylenetetramine-functionalized multi-walled carbon nanotube/silane-modified nano-sized silicon carbide, Compos. Part A Appl. Sci. Manuf. 41 (2010) 215-221.

[8] Y. Hwang, M. Kim, J. Kim, Improvement of the mechanical properties and thermal conductivity of poly(ether-ether-ketone) with the addition of graphene oxide-carbon 
nanotube hybrid fillers, Compos. Part A Appl. Sci. Manuf. 55 (2013) 195-202.

[9] M.R. Zakaria, H.M. Akil, M.H.A. Kudus, S.S.M. Saleh, Enhancement of tensile and thermal properties of epoxy nanocomposites through chemical hybridization of carbon nanotubes and alumina, Compos. Part A Appl. Sci. Manuf. 66 (2014) 109-116.

[10] W. Zhou, Q. Chen, X. Sui, L. Dong, Z. Wang, Enhanced thermal conductivity and dielectric properties of Al/??-SiCw/PVDF composites, Compos. Part A Appl. Sci. Manuf. $71(2015)$ 184-191.

[11] C.-W. Nan, G. Liu, Y. Lin, M. Li, Interface effect on thermal conductivity of carbon nanotube composites, Appl. Phys. Lett. 85 (2004) 3549.

[12] S. Hida, T. Hori, T. Shiga, J. Elliott, J. Shiomi, Thermal resistance and phonon scattering at the interface between carbon nanotube and amorphous polyethylene, Int. J. Heat Mass Transf. 67 (2013) 1024-1029.

[13] T. Evgin, H.D. Koca, N. Horny, A. Turgut, I.H. Tavman, M. Chirtoc, M. Omastová, I. Novak, Effect of aspect ratio on thermal conductivity of high density polyethylene/multiwalled carbon nanotubes nanocomposites, Compos. Part A Appl. Sci. Manuf. 82 (2016) 208-213.

[14] A. Tessema, D. Zhao, A. Kidane, S.K. Kumar, Effect of Micro-Cracks on the Thermal Conductivity of Particulate Nanocomposite, in: Fract. Fatigue, Fail. Damage Evol. Vol. 8, Springer, 2016: pp. 89-94.

[15] R. Kothari, C.T. Sun, R. Dinwiddie, H. Wang, Experimental and numerical study of the effective thermal conductivity of nano composites with thermal boundary resistance, Int. J. Heat Mass Transf. 66 (2013) 823-829.

[16] A. Tessema, A. Kidane, The Effect of Particles Size on the Thermal Conductivity of 
Polymer Nanocomposite, in: Compos. Hybrid, Multifunct. Mater. Vol. 4, Springer, 2015: pp. $151-156$.

[17] A. Rungta, B. Natarajan, T. Neely, D. Dukes, L.S. Schadler, B.C. Benicewicz, Grafting Bimodal Polymer Brushes on Nanoparticles Using Controlled Radical Polymerization, (2012).

[18] P. Akcora, S.K. Kumar, J. Moll, S. Lewis, L.S. Schadler, Y. Li, B.C. Benicewicz, A. Sandy, S. Narayanan, J. Ilavsky, P. Thiyagarajan, R.H. Colby, J.F. Douglas, "Gel-like" mechanical reinforcement in polymer nanocomposite melts, Macromolecules. 43 (2010) $1003-1010$.

[19] N. Jouault, D. Zhao, S.K. Kumar, Role of casting solvent on nanoparticle dispersion in polymer nanocomposites, Macromolecules. 47 (2014) 5246-5255.

[20] D. Zhao, D. Schneider, G. Fytas, S.K. Kumar, Controlling the Thermomechanical Behavior of Nanoparticle / Polymer Films, (2014) 8163-8173.

[21] H. Zhou, L. Yang, W. You, Rational Design of High Performance Conjugated Polymers for Organic Solar Cells, Macromolecules. 45 (2012) 607-632.

[22] M. Choudhury, S. Mohanty, S.K. Nayak, Effect of surface modification of aluminum nitride on electrical and thermal characterizations of thermosetting polymeric nanocomposites, Polym. Compos. 34 (2013) 1-14.

[23] H. Hu, L. Onyebueke, A. Abatan, Characterizing and Modeling Mechanical Properties of Nanocomposites- Review and Evaluation, J. Miner. Mater. Charact. Eng. 9 (2010) 275319.

[24] P.J. Lin, J.W. Ju, Effective elastic moduli of three-phase composites with randomly located and interacting spherical particles of distinct properties, Acta Mech. 208 (2009) 
11-26.

[25] M. Wang, N. Pan, Elastic property of multiphase composites with random microstructures, J. Comput. Phys. 228 (2009) 5978-5988.

[26] Z. Han, A. Fina, Thermal conductivity of carbon nanotubes and their polymer nanocomposites: A review, Prog. Polym. Sci. 36 (2011) 914-944. 
Figures

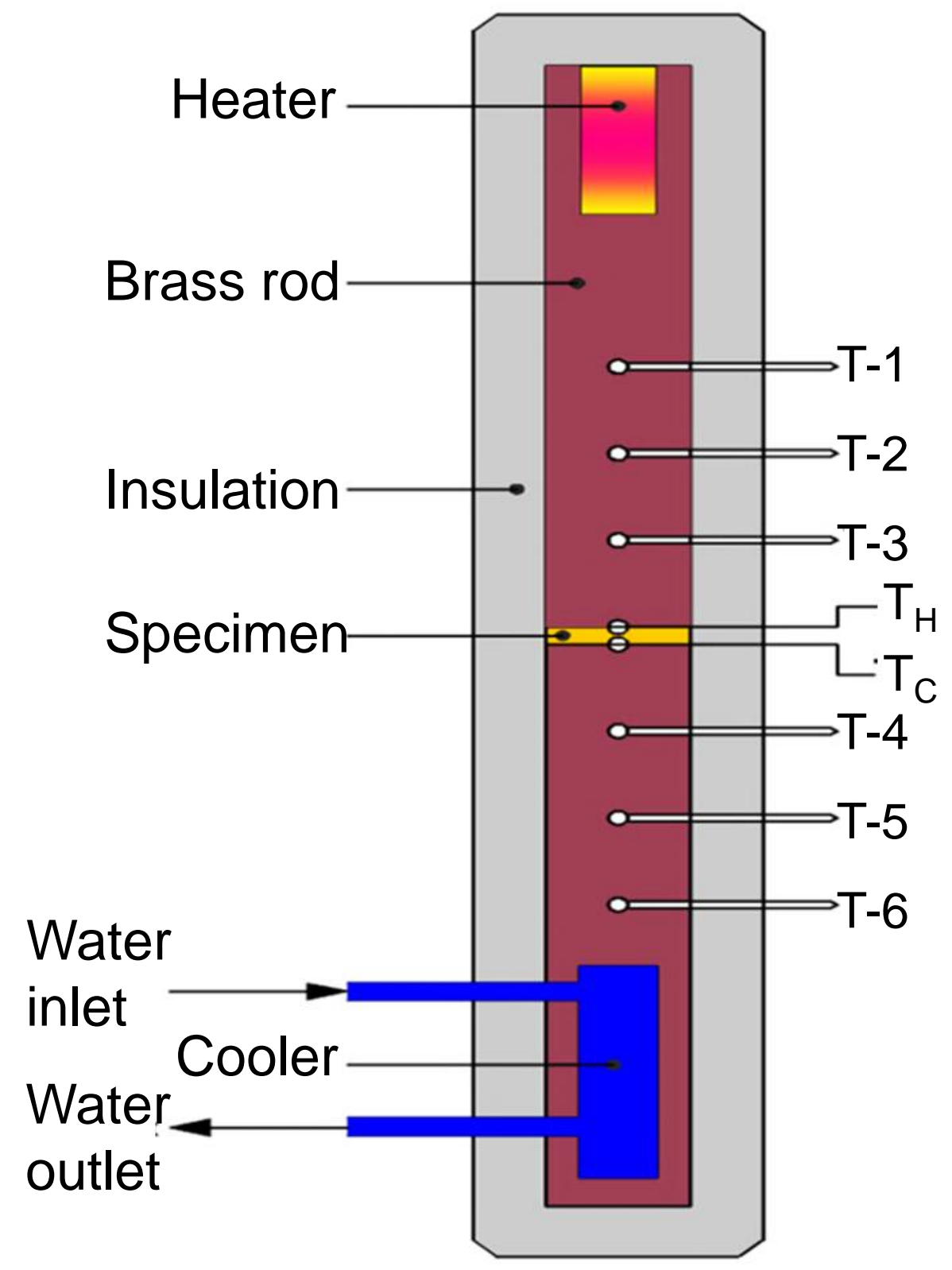

Figure 1: Schematic diagram of unidirectional/linear heat transfer device. T1-T8 represents the locations of the eight thermocouples, respectively. 


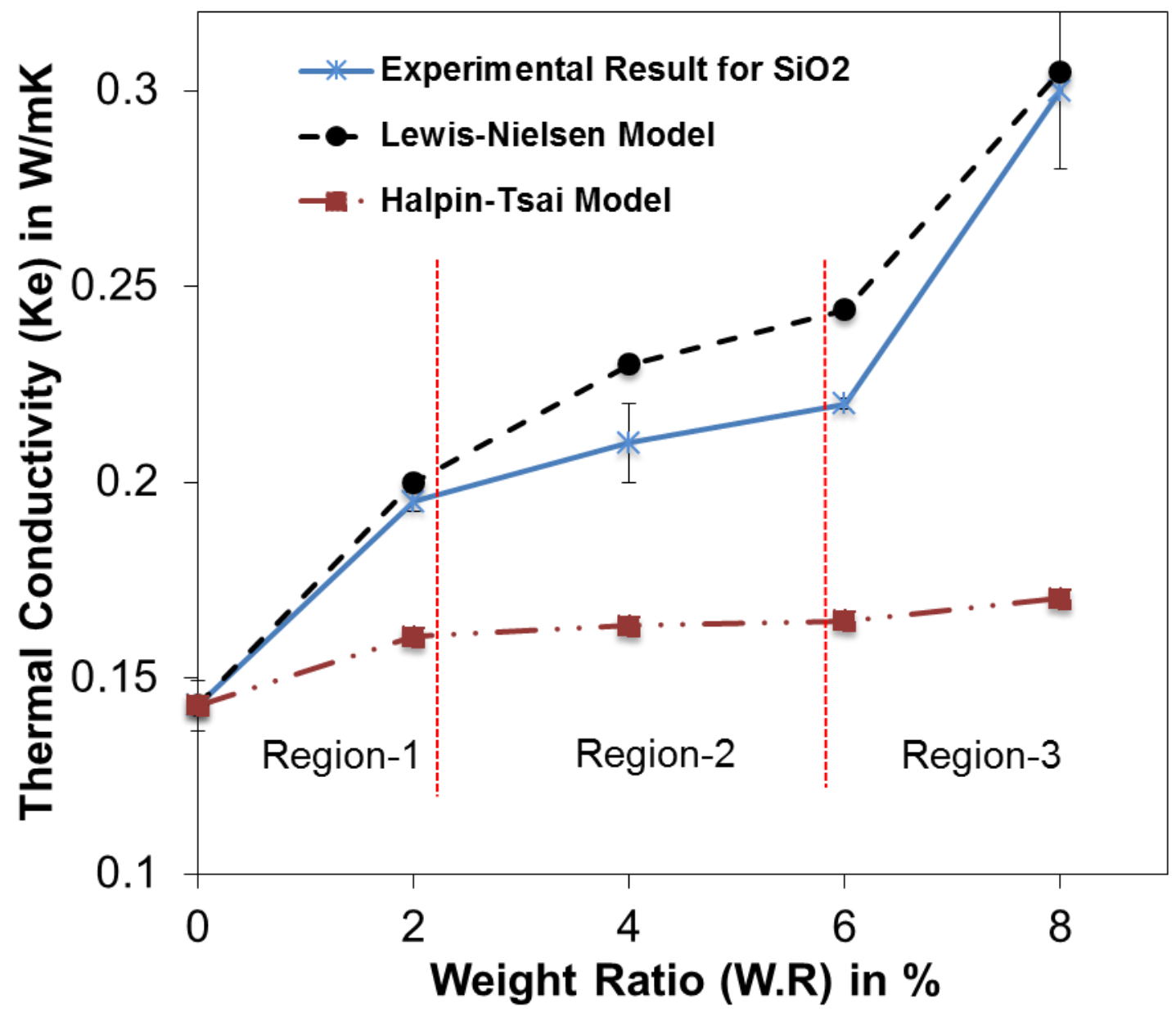

Figure 2: The effective thermal conductivity $(K e)$ of $\mathrm{SiO}_{2}$-epoxy nanocomposites (Type-1) as a function of silica weight fraction. 


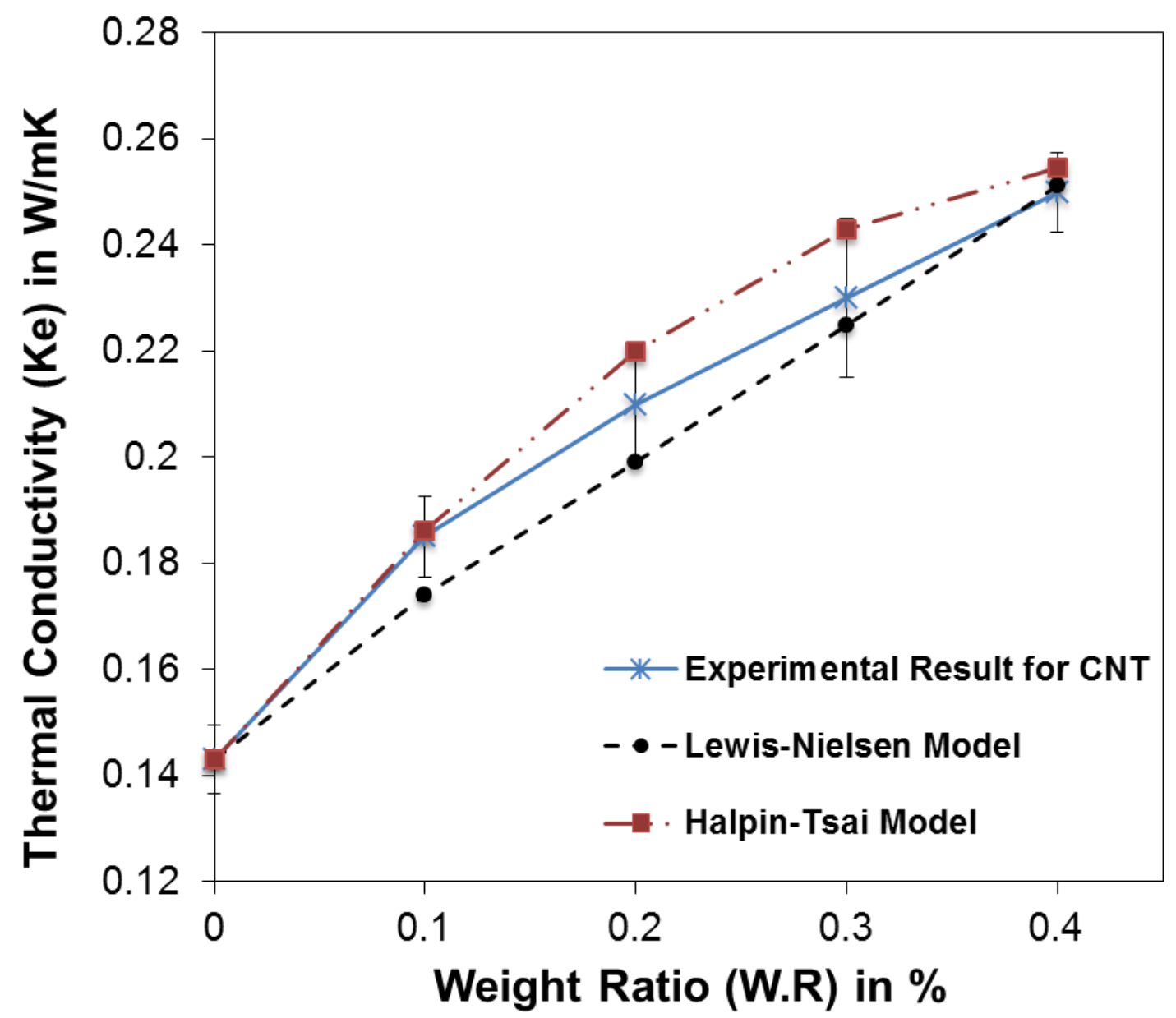

Figure 3: The effective thermal conductivity $(\mathrm{Ke})$ of CNT-epoxy nanocomposites (Type-2) as a function of particle weight fraction. 

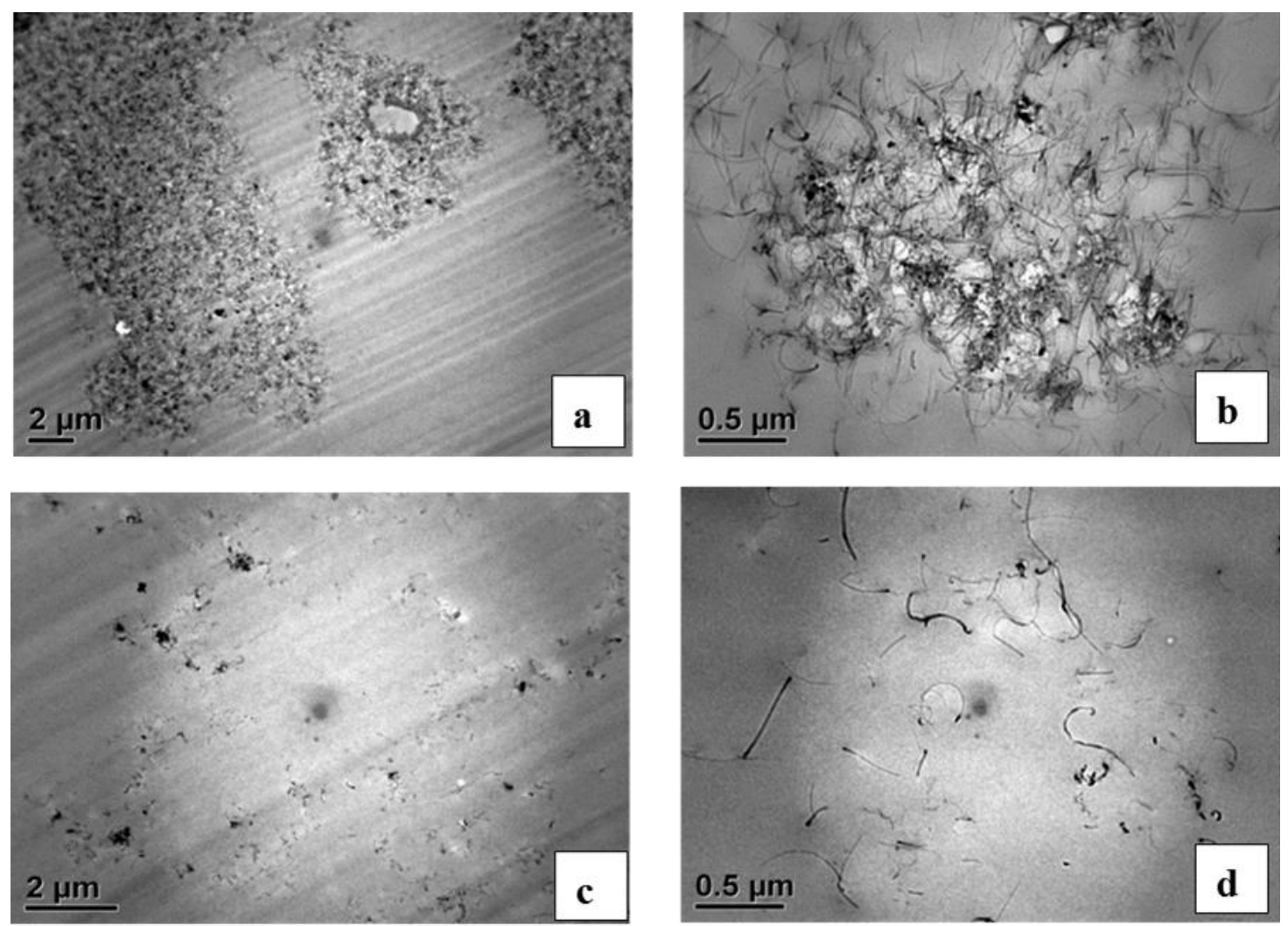

Figure 4: TEM images of non-ultrasonicated (a and b) and ultrasonicated (c and $\mathbf{d})$ naocomposites for $0.2 \mathrm{wt} \% \mathrm{CNT}$ samples.
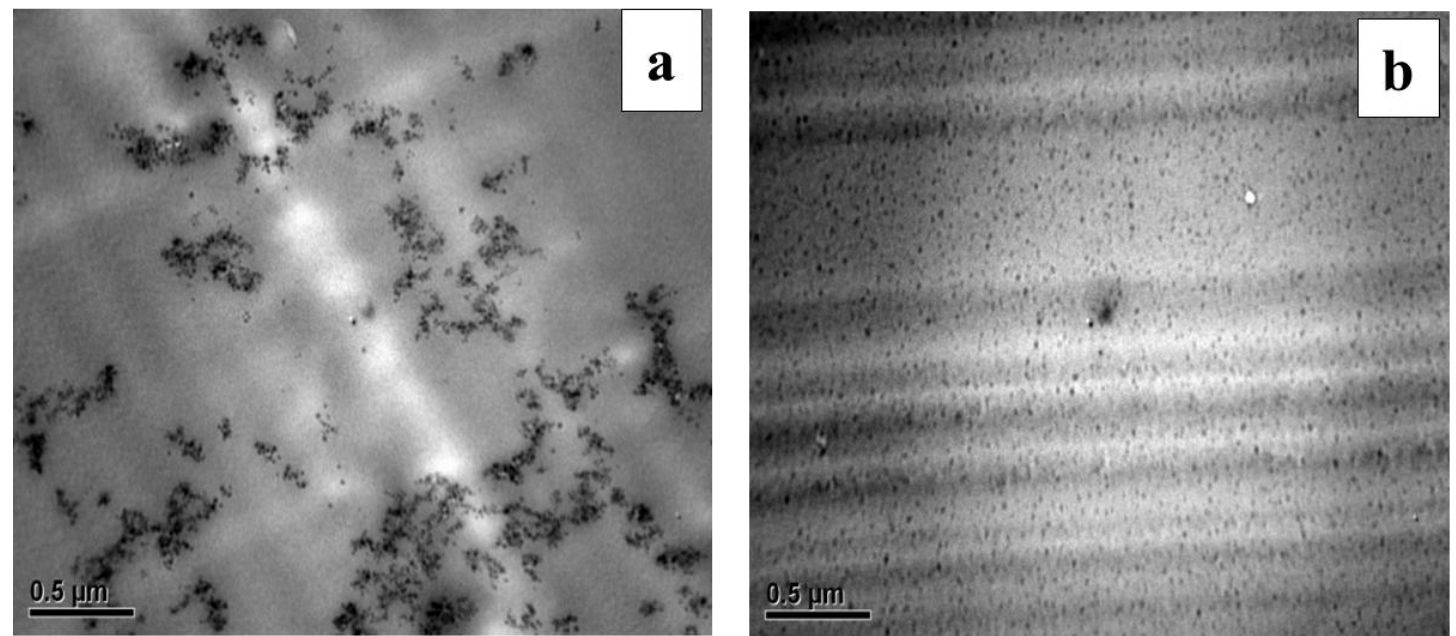

Figure 5: TEM images of P2VP matrices filled with 5 wt \% silica NPs solvent cast from (a) pyridine (b) MEK. The samples had been thermally annealed for $24 \mathrm{~h}$ at $150^{\circ} \mathrm{C}$ under vacuum. 


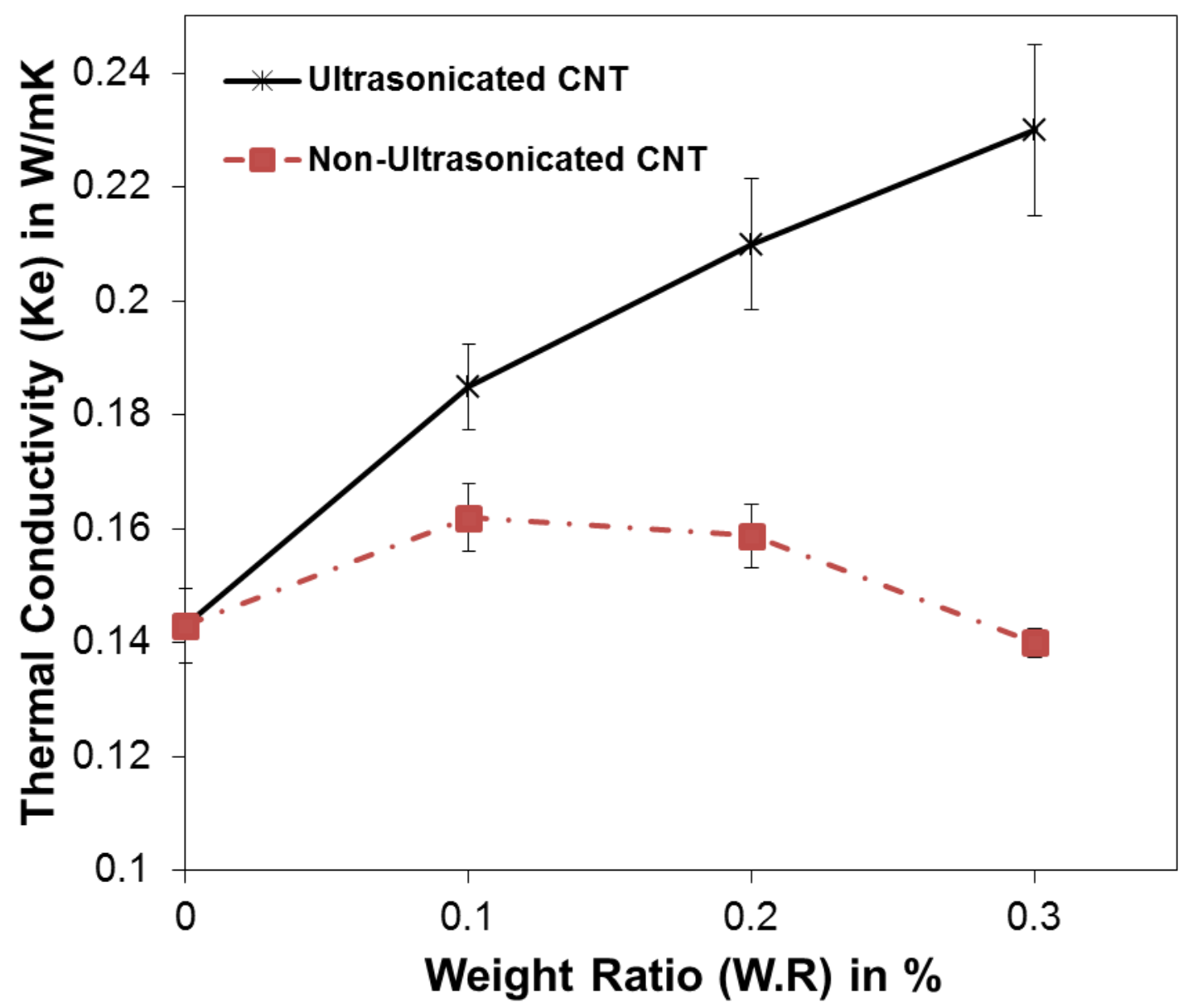

Figure 6: Variation of Effective Thermal Conductivity with weight ratio for Ultrasonicated and Non-ultrasonicated CNT composite at $30{ }^{\circ} \mathrm{C}$. 


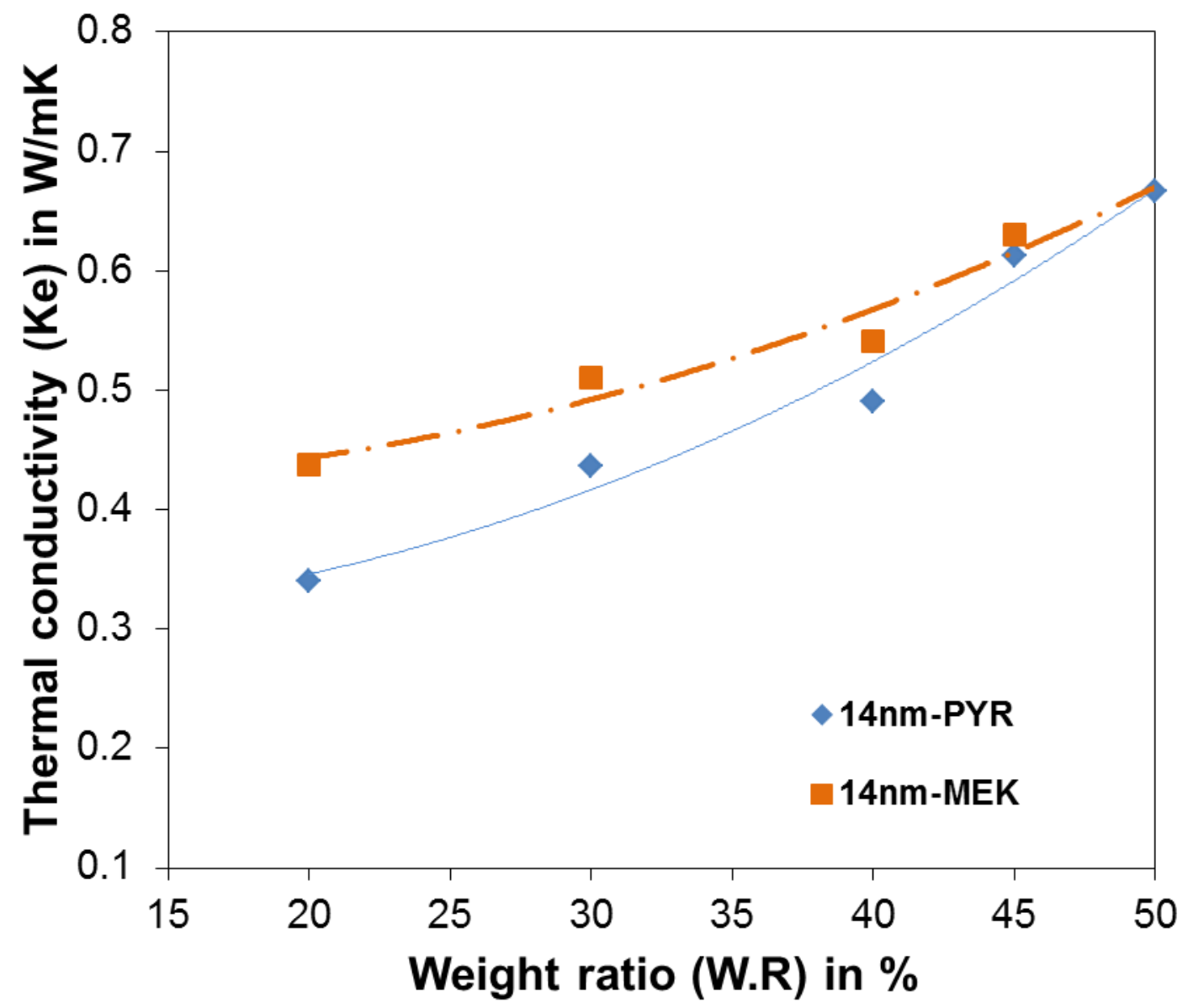

Figure 7: Variation of Effective Thermal Conductivity with weight ratio for $\mathrm{SiO}_{2}-\mathrm{P} 2 \mathrm{VP}$ composite with solvent pyridine and MEK. 


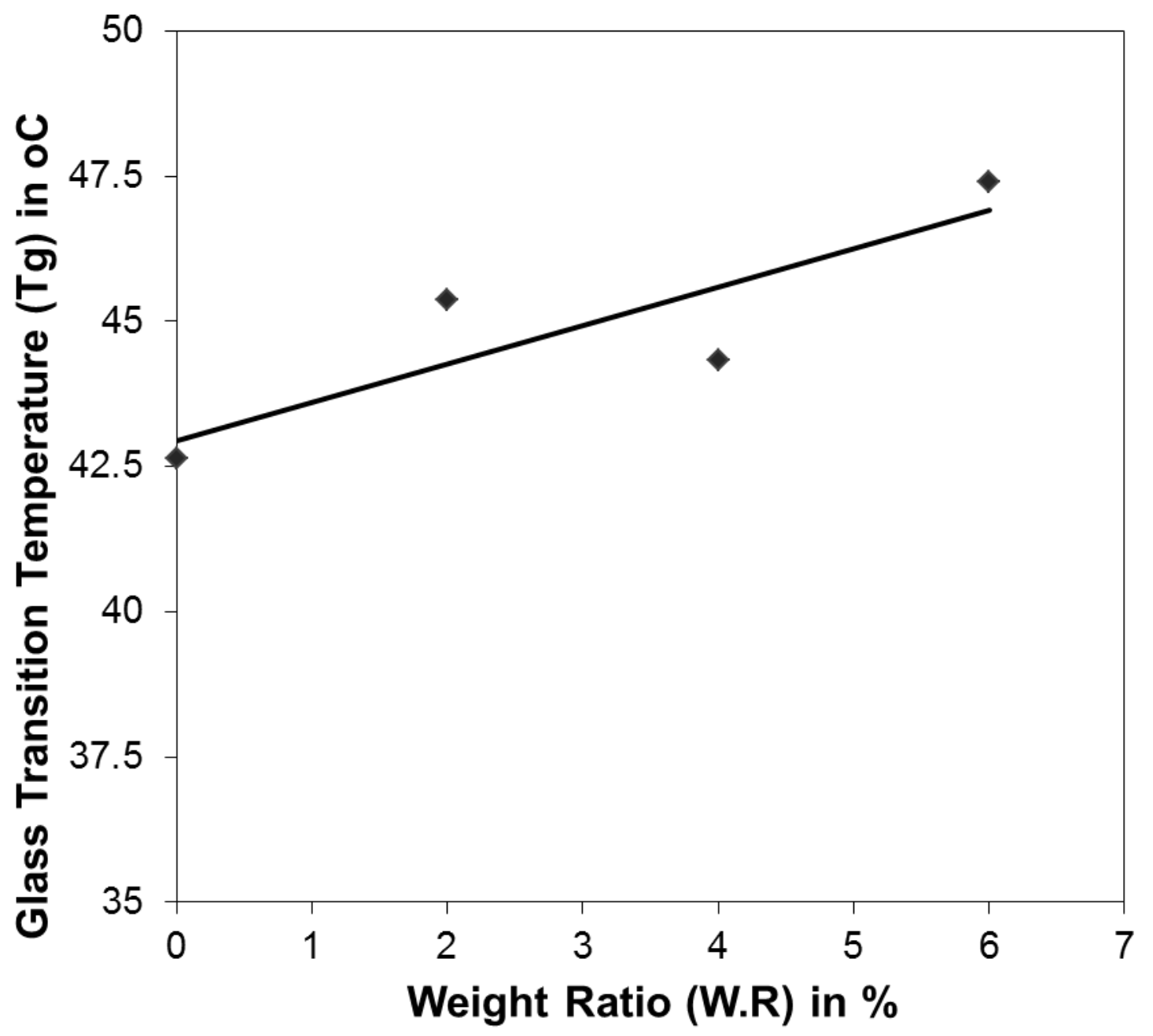

Figure 8: Glass transition temperature (Tg) of silica composite (Type-1) for different weight ratio. 


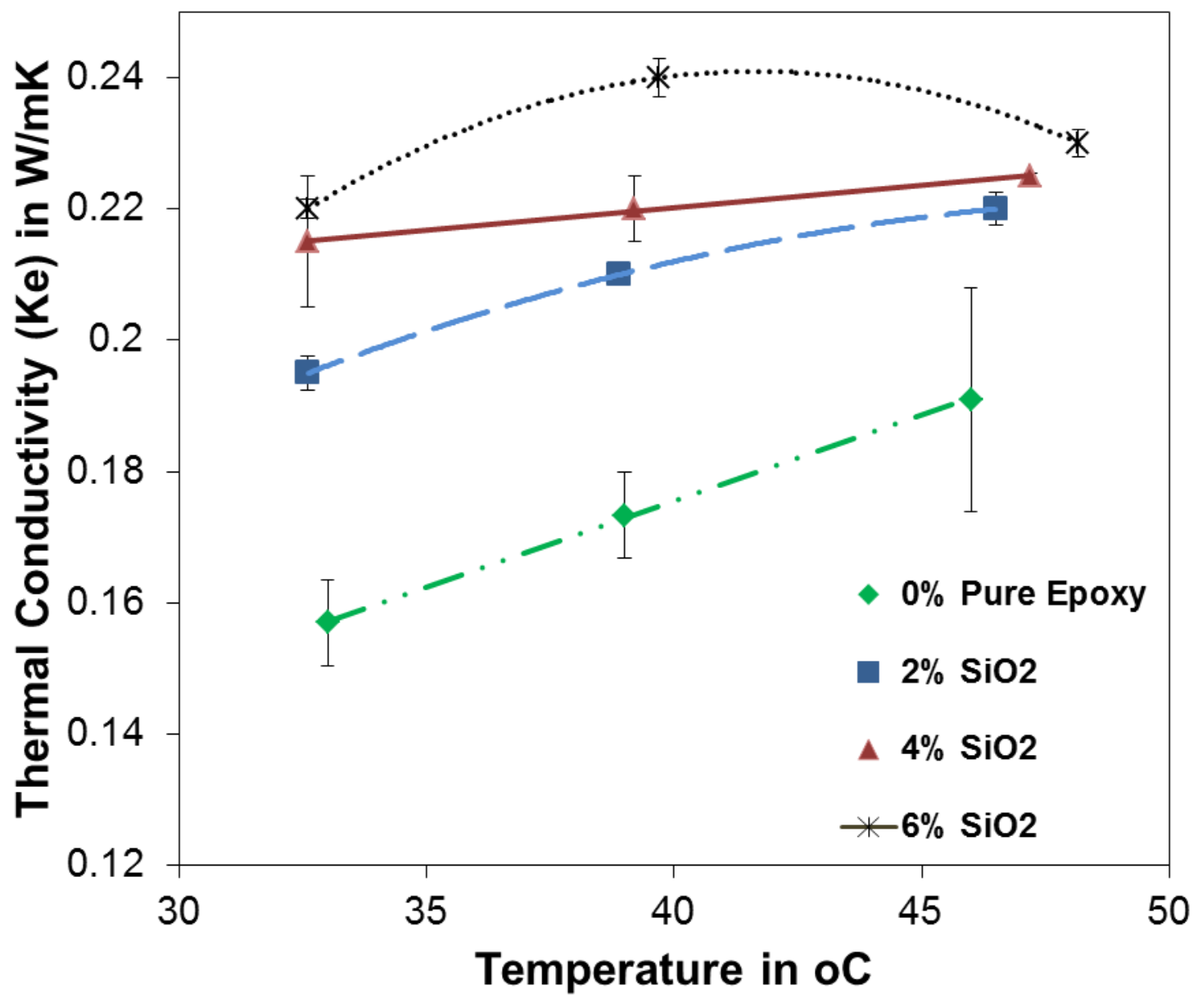

Figure 9: Variation of Ke with Temperature (T) for different weight ratio of Silica composites. 